\title{
A Hamiltonian Approach to the Mass of Isolated Black Holes
}

\author{
Christopher Beetle and Stephen Fairhurst \\ Center for Gravitational Physics and Geometry \\ Department of Physics, The Pennsylvania State University \\ University Park, PA 16802
}

\begin{abstract}
Boundary conditions defining a non-rotating isolated horizon are given in Einstein-Maxwell theory. A spacetime representing a black hole which itself is in equilibrium but whose exterior contains radiation admits such a horizon. Inspired by Hamiltonian mechanics, a (quasi-)local definition of isolated horizon mass is formulated. Although its definition does not refer to infinity, this mass takes the standard value in a Reissner-Nordström solution. Furthermore, under certain technical assumptions, the mass of an isolated horizon is shown to equal the future limit of the Bondi energy.
\end{abstract}

\section{INTRODUCTION}

In their standard form, the zeroth and first laws of black hole mechanics apply only to the relatively small class of stationary black hole solutions. These solutions seem too limited to describe physically realistic situations since the requirement of stationarity precludes the presence of radiation even far from the horizon. As an example, consider the gravitational collapse depicted in figure 1. Although the horizon will presumably reach equilibrium at late times, there will also be gravitational and other radiation near null infinity. One would hope the familiar laws of black hole mechanics continue to apply in such situations. Recently, using the framework of isolated horizons [1,2], this expectation has been shown to be correct: The zeroth and first laws extend to a broad class of spacetimes containing both radiation and black holes which are, however, isolated from that radiation. This contribution focuses on the definition of black hole mass in this expanded, non-stationary setting and collects the results presented in both of our talks at the Conference.

The key idea of the isolated horizon framework is to replace the global construct of an event horizon with a set of boundary conditions applied locally at the surface of the black hole. These boundary conditions model a portion of the horizon which is isolated, i.e., across which there is no flux of gravitational radiation or matter. However, since the boundary conditions are applied only at the horizon, the exterior of an isolated horizon will generically contain both. For technical simplicity, we confine our attention here to non-rotating isolated horizons in Einstein-Maxwell theory.

To formulate the laws of black hole mechanics for isolated horizons, one needs definitions of the "extrinsic parameters" of a black hole - particularly its mass $M$ and surface gravity $\kappa$ - which depend only on the structure available at the horizon. In the static context, $M$ is taken to be the ADM mass and $\kappa$ to be the acceleration at the event horizon of that static Killing field which is unit at infinity. Although these parameters are associated with the black hole, they cannot be constructed solely from the near-horizon geometry; they are genuinely global concepts. In the non-static context, the ADM energy cannot be identified with the mass of the black hole since it will also include contributions from the energy contained in radiative fields far from the horizon. Similarly, without a static Killing field, the usual prescription for normalizing the null generator of the horizon is no longer applicable and the usual definition of $\kappa$ therefore fails. Drawing motivation from the Hamiltonian formulation of an isolated horizon, this paper shows how the problem of defining its mass can be overcome.

The details of our construction, along with a more complete set of references, may be found in [2]. 


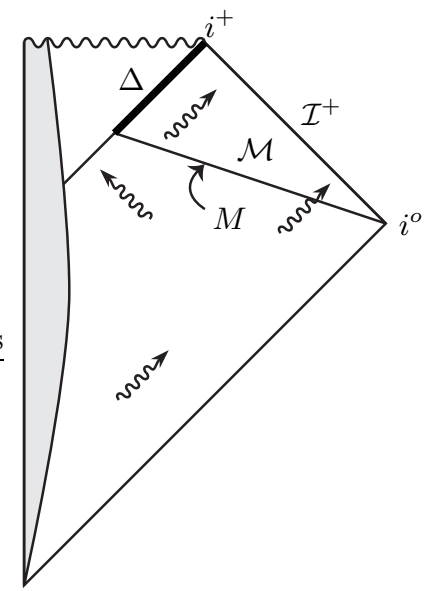

FIGURE 1. A typical gravitational collapse. The portion $\Delta$ of the horizon at late times is isolated. The spacetime $\mathcal{M}$ of interest is the triangular region bounded by $\Delta, \mathcal{I}^{+}$and a partial Cauchy slice $M$.

\section{ISOLATED HORIZONS}

An isolated horizon is defined by a set of boundary conditions which capture the essential local structure of a stationary event horizon. The physical situation we wish to model is illustrated by the example of figure 1 . The late stages of the collapse pictured here should describe a non-dynamical, isolated black hole. However, a realistic collapse will generate gravitational radiation which must either be scattered back into the black hole or radiated to infinity. Physically, one expects most of the back-scattered radiation will be absorbed rather quickly and, in the absence of outside perturbations, the black hole will "settle down" to a steady-state configuration. Although the presence of radiation elsewhere in spacetime implies $\mathcal{M}$ cannot be stationary, the portion $\Delta$ of the event horizon at late times will describe an isolated black hole. The boundary conditions are designed to represent precisely such situations. They are also satisfied at the event horizon of a static, Reissner-Nordström black hole.

We are now in a position to state our definition. For clarity, we shall give a fairly complete statement of the boundary conditions, even though some of the details will not be needed here. A non-rotating, isolated horizon is a three-dimensional hypersurface $\Delta$ in spacetime which satisfies the following four conditions:

(a) $\Delta$ is null, topologically $S^{2} \times \mathrm{R}$, and equipped with a preferred foliation by two-spheres $S_{\Delta}$ transverse to its null normal $\ell^{a}$. We will denote the second null normal to the foliation by $n^{a}$ and partially fix the normalizations by setting $\ell^{a} n_{a}=-1$ and requiring the pull-back to $\Delta$ of $n_{a}$ to be curl-free.

(b) $\Delta$ is a non-rotating, non-expanding, future boundary of spacetime. Specifically, $\ell^{a}$ is twist-, shear- and expansion-free and $n^{a}$ is twist- and shear-free with strictly negative expansion $\theta_{(n)}$ which is constant on each spherical leaf of the preferred foliation of $\Delta .^{1}$

(c) All equations of motion are satisfied at $\Delta$.

(d) The flux densities of the electric and magnetic fields are both constant on each two-sphere $S_{\Delta}$.

Let us summarize the consequences of the boundary conditions which are directly relevant here.

First, the notion of an isolated horizon is intrinsically local since it is defined through a set of boundary conditions. This makes the definition well-suited to situations such as that of figure 1. Although the boundary conditions imply the surface $\Delta$ is isolated from infalling matter and radiation, the geometry of the exterior region $\mathcal{M}$ remains largely unconstrained. Even at $\Delta$, the outgoing radiative modes (i.e., those flowing along $\Delta)$ are undetermined.

1) The spherical symmetry of $\theta_{(n)}$ guarantees the uniqueness of the preferred foliation. 
Second, a non-rotating isolated horizon in Einstein-Maxwell theory is characterized by three parameters; its radius $r_{\Delta}$, electric charge $Q_{\Delta}$ and magnetic monopole moment $P_{\Delta}$. Much of the intrinsic structure of $\Delta$ depends only on this remarkably small set of parameters. In particular, the Newman-Penrose components $\Psi_{2}$ and $\phi_{1}[3]$, describing respectively the "Coulombic parts" of the gravitational and electromagnetic fields at $\Delta$, are functions of these variables alone.

Third, the boundary conditions guarantee all three parameters are constant in time. The radius is timeindependent due to the vanishing expansion of $\Delta$ and the imposition of the Maxwell equations at $\Delta$ implies the same for the electromagnetic charges. It is important to note that this time-independence is a result of boundary conditions and not of dynamics. Consequently, this result holds in any history compatible with the boundary conditions and not just "on-shell."

Before moving on to our discussion of mass, let us briefly consider the surface gravity. As discussed in the Introduction, the standard formulation of black hole mechanics defines surface gravity as the acceleration of the appropriately normalized null generator of the horizon. This normalization is possible since the horizongenerating Killing field can be fixed to an unit time translation at infinity. However, a generic isolated horizon spacetime possesses no such Killing field. To overcome this problem, we must provide a means of fixing the normalization of $\ell^{a}$ on any isolated horizon; then $\kappa$ may again be defined as the acceleration of the properly normalized $\ell^{a}$. Recall that $\ell^{a}$ itself is null and free of shear, expansion and twist, whence we cannot normalize it through its intrinsic properties. However, since $\theta_{(n)}$ is strictly negative by condition (b), we can normalize $n^{a}$ by setting its expansion to some given value. This in turn will fix the normalization of $\ell^{a}$ since $\ell^{a} n_{a}=-1$.

Fortunately, a preferred value of $\theta_{(n)}$ exists. In a Reissner-Nordström solution, with $\ell^{a}$ taken to be the restriction to $\Delta$ of the properly normalized static Killing field, one finds $\theta_{(n)}=-2 / r_{\Delta}$. Since we want to include this family of solutions in our analysis, let us require $n^{a}$ (and hence $\ell^{a}$ ) always be normalized such that $\theta_{(n)}=-2 / r_{\Delta}$. This convention, which is not a consequence of the boundary conditions, provides a definition of surface gravity for a generic isolated horizon: The value of $\kappa$ is simply the acceleration of the properly normalized $\ell^{a}$.

\section{THE HAMILTONIAN AND BLACK HOLE MASS}

A key question faced by any set of boundary conditions is whether they allow the formulation of a welldefined action principle. For boundary conditions enforcing asymptotic flatness in spacetimes with no interior boundaries, it is well known that an action principle can be found by adding a boundary term at infinity to the standard bulk action. The situation is similar when one allows an isolated horizon at an interior boundary. A well-defined action principle can then be obtained by also including a boundary term at $\Delta$ [4]. However, as we will see now, there is some subtlety in using this action principle to formulate a Hamiltonian description of an isolated horizon system.

In passing to the Hamiltonian framework, one performs a Legendre transform of the action. To do so, let us foliate the spacetime $\mathcal{M}$ with partial Cauchy surfaces $M$ which extend to spatial infinity and whose inner boundaries are the preferred two spheres $S_{\Delta}$. Next, fix a smooth vector field $t^{a}$, transverse to the leaves $M$, which tends to a $\ell^{a}$ at the horizon ${ }^{2}$ and to an unit time translation orthogonal to $M$ near infinity. Then, after the Legendre transform, the Hamiltonian corresponding to evolution along $t^{a}$ can be written in the form

$$
H_{t}=\int_{M}(\text { constraints })+H_{\infty}-H_{\Delta}
$$

The quantities $H_{\infty}$ and $H_{\Delta}$ represent the surface terms in the Hamiltonian at infinity and $\Delta$ respectively. The term at infinity is the usual one arising from the asymptotically flat boundary conditions imposed there. It can be expressed in terms of components of the Weyl curvature as

$$
H_{\infty}=\lim _{R \rightarrow \infty} \oint_{S_{R}}\left[-\frac{R}{4 \pi G} \Psi_{2}\right]{ }^{2} \epsilon
$$

On any solution to the field equations, this surface term will equal the ADM energy of spacetime.

2) Here, $\ell^{a}$ denotes the null generator of $\Delta$ normalized according to the prescription given at the end of the previous section. Physically, this vector field defines the "rest frame" of the black hole. 
On the other hand, due to an ambiguity in the action caused by the variational principle, the horizon boundary term $H_{\Delta}$ in the Hamiltonian is initially an arbitrary function of the parameters $r_{\Delta}, Q_{\Delta}$ and $P_{\Delta}$. Since every allowable history must satisfy our boundary conditions, the variation of these parameters must be time-independent. However, the action principle allows only variations which vanish at the initial and final time slices. It follows that $\delta r_{\Delta}, \delta Q_{\Delta}$ and $\delta P_{\Delta}$ vanish everywhere in the Lagrangian formalism. Therefore, one may add any function of these parameters to the action and still have a well-posed variational principle. As a result of this freedom, $H_{\Delta}$ will not be fixed by the Lagrangian.

The ambiguity in $H_{\Delta}$ is resolved within the Hamiltonian formalism. The reason for this lies in subtle differences between the Lagrangian and Hamiltonian variations. In the Hamiltonian framework, the phase space consists of fields on a fixed spacelike three-manifold $M$ which describe horizons of arbitrary radius and charges. Consequently, there are tangent vectors $\delta$ to phase space which change the values of $r_{\Delta}, Q_{\Delta}$ and $P_{\Delta}$. Requiring the consistency of Hamilton's equations under such variations in phase space determines the Hamiltonian uniquely. The resulting horizon surface term in the Hamiltonian is

$$
H_{\Delta}=\oint_{S_{\Delta}}\left[-\frac{r_{\Delta}}{4 \pi G} \Psi_{2}+\frac{Q_{\Delta}-i P_{\Delta}}{2 \pi r_{\Delta}} \phi_{1}\right]{ }^{2} \epsilon
$$

where the Newman-Penrose components $\Psi_{2}$ and $\phi_{1}$ are determined in terms of $r_{\Delta}, Q_{\Delta}$ and $P_{\Delta}$ by the boundary conditions.

Remarkably, in a static Reissner-Nordström solution, one finds the horizon surface term (3) is exactly equal to that at infinity (2). Consequently, the full Hamiltonian vanishes in a static solution. This feature is not accidental: There is a general argument from symplectic geometry which requires the vanishing of $H_{t}$ on a stationary solution.

Now let us turn to the definition of black hole mass. In physical theories, energy is defined as the on-shell value of the Hamiltonian generating an appropriate time translation. In general relativity, the bulk term in the Hamiltonian consists solely of constraints and therefore vanishes on-shell. Thus, the energy of a system is given by the surface terms in its Hamiltonian. For example, in asymptotically flat spacetime, the Hamiltonian surface term at infinity is precisely equal to the ADM energy when the constraints are satisfied. In analogy, we define the mass of an isolated horizon to be the horizon surface term in the Hamiltonian:

$$
M_{\Delta}:=H_{\Delta}=\oint_{S_{\Delta}}\left[-\frac{r_{\Delta}}{4 \pi G} \Psi_{2}+\frac{Q_{\Delta}-i P_{\Delta}}{2 \pi r_{\Delta}} \phi_{1}\right]{ }^{2} \epsilon .
$$

This formula for black hole mass resembles the expression (2) for ADM mass, but includes an additional contribution from the electromagnetic field. Without this contribution, $M_{\Delta}$ would not give the correct mass for a Reissner-Nordström black hole. For a generic isolated horizon, the expression (4) for $M_{\Delta}$ can be regarded as "the mass of the black hole together with its Coulombic hair." That is, it includes the energy associated with static fields emanating from $\Delta$, but not contributions due to radiative excitations outside $\Delta$.

\section{RADIATIVE ENERGY AND BLACK HOLE MASS}

As discussed in the Introduction, the ADM energy cannot be used to measure the mass of a non-stationary black hole since it also includes contributions from the radiative modes of the fields. This raises the question of whether there exists a precise relation among the ADM energy, the black hole mass and the radiative energy. One would expect such a relation to be simply

$$
(\mathrm{ADM} \text { energy })=(\text { black hole mass })+(\text { radiative energy })
$$

We will now show that, under certain technical assumptions, this relation holds within the framework of isolated horizons. This result is on a somewhat different footing from the rest of the calculations presented here due to the additional assumptions needed to complete the proof. Nevertheless, from a physical point of view, the relation (5) serves to strengthen our intuition regarding isolated horizon mass and provides further justification for our definition.

Consider an isolated horizon, such as $\Delta$ in figure 1, which extends to future time-like infinity $i^{+}$. Since no radiation can cross $\Delta$, all the radiation in $\mathcal{M}$ must "register on" future null infinity $\mathcal{I}^{+}$. It is therefore not surprising that the radiative data can be encoded in fields on $\mathcal{I}^{+}$[5]. Furthermore, these fields on $\mathcal{I}^{+}$admit a 
phase space structure [6]. That is, there exists a symplectic structure $\Omega_{\mathcal{I}}$ on the space of data at null infinity and a Hamiltonian $H_{\mathcal{I}}$ which generates time evolution in that space. Moreover, on any solution to the field equations, the value of $H_{\mathcal{I}}$ is precisely equal to the total radiative energy. This fact is the key to the proof of (5) for isolated horizon systems.

We now have two phase spaces; the isolated horizon phase space $\left(\Omega, H_{t}\right)$ discussed in the previous section and the asymptotic phase space $\left(\Omega_{\mathcal{I}}, H_{\mathcal{I}}\right)$ introduced here. Both of these describe the radiative modes of the fields in $\mathcal{M}$, and hence it is reasonable to expect they should also be "equivalent" in some appropriate sense. This expectation turns out to be correct. There is a natural map from the isolated horizon phase space to the phase space at null infinity such that $\Omega \mapsto \Omega_{\mathcal{I}}$. Using this fact, we can apply Hamilton's equations in each phase space to find

$$
\delta H_{t}=\Omega\left(\delta, X_{H}\right)=\Omega_{\mathcal{I}}\left(\delta, X_{H}\right)=\delta H_{\mathcal{I}} .
$$

It follows that the isolated horizon Hamiltonian and its counterpart at null infinity differ at most by a constant. In the previous section, however, we showed the isolated horizon Hamiltonian $H_{t}$ vanishes on a ReissnerNordström solution. Furthermore, these static solutions contain no radiation, whence the Hamiltonian at $\mathcal{I}^{+}$ must also vanish. Therefore, the "constant of integration" for (6) is zero and the two Hamiltonians are equal on any solution to the equations of motion. Since $H_{t}$ equals the difference between the ADM energy and the black hole mass by (1) and $H_{\mathcal{I}}$ equals the radiative energy in spacetime, the relation (5) follows.

Therefore, the mass of an isolated horizon is equal to the ADM energy of spacetime minus the energy contained in radiation outside the horizon. It is well known that the difference of the ADM energy and the flux of energy through $\mathcal{I}^{+}$is the future limit of the Bondi energy. Hence, isolated horizon mass is the future limit of the Bondi energy. Equivalently, $M_{\Delta}$ can be thought of as the mass remaining in spacetime after all radiation has escaped to infinity.

\section{SUMMARY}

We have presented the definition of a non-rotating isolated horizon. Within the surrounding framework, we have shown how the definitions of mass (and surface gravity) can be extended to a non-static context. Although it has not been discussed here, these definitions enable one to formulate and prove the zeroth and first laws of black hole mechanics for generic isolated horizons [2].

The mass $M_{\Delta}$ is given by the horizon surface term in the unique consistent Hamiltonian for an isolated horizon system. It is manifestly (quasi-)local to the horizon and correctly reproduces the mass of ReissnerNordström black holes. Furthermore, given certain technical assumptions, one can show the ADM energy of a spacetime containing a single isolated horizon is precisely the sum of $M_{\Delta}$ and the total energy contained in the radiative modes of the fields. In this case, $M_{\Delta}$ is equal to the future limit of the Bondi energy.

Acknowledgements We would like to express our gratitude to Abhay Ashtekar for his collaboration in the work reported here. The authors were supported in part by NSF grants PHY95-14240 and INT97-22514 and by the Eberly research funds of the Pennsylvania State University.

\section{REFERENCES}

1. A. Ashtekar, A. Corichi and K. Krasnov. Isolated horizons: the classical phase space. Adv. Theor. Math. Phys. in press; gr-qc/9905089.

2. A. Ashtekar, C. Beetle and S. Fairhurst. Isolated horizons: a generalization of black hole mechanics. Class. Quantum Grav. 16 (1999) L1-L7.

A. Ashtekar, C. Beetle and S. Fairhurst. Mechanics of isolated horizons. Penn State preprint; gr-qc/9907068.

3. R. Penrose and W. Rindler. Spinors and Spacetime, Volume 1. Cambridge University Press, Cambrindge, 1984.

4. A. Ashtekar, J. Baez, A. Corichi and K. Krasnov. Quantum geometry and black hole entropy. Phys. Rev. Lett. 80 (1998) 904-907.

5. A. Ashtekar. Radiative degrees of freedom of the gravitational field in exact general relativity. J. Math. Phys. 22 (1981) 2885-2895.

6. A. Ashtekar and M. Streubel. Symplectic geometry of radiative modes and conserved quantities at null infinity. Proc. R. Soc. Lond. A 376 (1981) 585-607. 